\title{
Improvement of Arbutin Trans-Epidermal Delivery Using Radiofrequency Microporation
}

\author{
Junho Won and Jin Woo Park* \\ College of Pharmacy and Natural Medicine Research Institute, Mokpo National University, 1666 Youngsan-ro, Muan-gun, \\ Jeonnam 534-729, Republic of Korea \\ *For correspondence: Email: jwpark@mokpo.ac.kr; Tel: +82-61-450-2704; Fax: +82-61-450-2689
}

\begin{abstract}
Purpose: To assess the ability of radiofrequency (RF) microporation to promote trans-epidermal delivery of arbutin.

Methods: To investigate the enhancing effect of RF microchannels on skin permeation of arbutin, in vitro skin permeability studies were performed with RF microporation-treated Hartley albino guinea pig skin using Franz diffusion cell system. Furthermore, improved depigmentation effects in brown guinea pig in vivo after treatment with RF microporation was evaluated to create hydrophilic microchannels for arbutin trans-epidermal delivery.

Results: RF microporator caused disruption of the stratum corneum (SC) and created $50 \mu \mathrm{m}$ diameter microchannels at a depth of $100 \mu \mathrm{m}$ in the skin. RF microporation increased skin permeability of arbutin 2.25-fold compared to untreated skin exposed to RF in vitro. Arbutin administration led to considerable skin depigmentation following RF microporation of UV-induced hyperpigmented skin in a 28-day in vivo experiment ( $\triangle L$-value on day 28: UV, $0.17 \pm 0.50 ; U V+R F, 0.07 \pm 0.48 ; U V+$ arbutin, $1.32 \pm 0.51$ and $U V+R F+$ arbutin, $1.82 \pm 0.53)$.

Conclusion: RF microporation seems to be a suitable physical trans-epidermal delivery technology for topical application of highly hydrophilic depigmentation agents through the hydrophobic skin barrier.
\end{abstract}

Keywords: Radiofrequency microporation, Trans-epidermal delivery, Depigmentation, Arbutin

Tropical Journal of Pharmaceutical Research is indexed by Science Citation Index (SciSearch), Scopus, International Pharmaceutical Abstract, Chemical Abstracts, Embase, Index Copernicus, EBSCO, African Index Medicus, JournalSeek, Journal Citation Reports/Science Edition, Directory of Open Access Journals (DOAJ), African Journal Online, Bioline International, Open-J-Gate and Pharmacy Abstracts

\section{INTRODUCTION}

Trans-epidermal delivery of hydrophilic, charged molecules is restricted due to the highly lipophilic barrier function of the stratum corneum (SC), the outermost dermal layer of the skin [1]. Microporation, which employs physical energy such as radiofrequency (RF), laser, iontophoresis, and ultrasound waves, has been introduced to enhance skin permeation by macromolecules or hydrophilic agents such as hormones, peptides, vaccines, DNA, and small interfering RNA [2-5]. A type of microporation using microneedles composed of a plurality of micro-projection arrays of different shapes and a base support can create micropores using mechanical pressure. However, the irregularity and elasticity of the skin surface may form heterogeneous microchannels and microneedles can break during application [6]. RF microporation technology was initially developed to remove abnormal tissue during surgery [7]. The current emitted from RF devices creates ablative microchannels in the skin by vibrating and vaporizing intracellular water molecules, resulting in the collapse of abnormal cellular structures and subsequent healing $[8,9]$. Water that leaks from collapsed tissue following RF 
microporation can form hydrophilic pathways for highly hydrophilic molecules to reach the lower epidermal layer or superficial dermis. Capitalizing on this phenomenon, several authors have attempted to increase transdermal drug delivery of macromolecules with biological activities $[10,11]$.

Arbutin is a glycosylated hydroquinone from the Arctostaphylos bearberry plant [12], which has been used widely as an effective skin-lightening agent that prevents melanin biosynthesis by inhibiting oxidation of tyrosine and L-3,4dihydroxyphenylalanine [13]. However, arbutin does not penetrate readily through the hydrophobic skin barrier due to its high hydrophilicity, limiting its effective concentration in the stratum basale, where melanocytes reside. Chemicals such as surfactants or alcohols have been applied to temporarily remove the SC to enhance arbutin trans-epidermal delivery but few have succeeded in delivering a sufficient amount of the compound [14]. In addition, no study has addressed the applicability of RF microporation for topical delivery of a small hydrophilic molecule such as arbutin, whose active site is restricted mainly to the dermis layer.

In this study, we demonstrated that RF microporation promoted trans-epidermal delivery of arbutin in Hartley albino guinea pig skin in vitro, and the enhanced skin permeability of arbutin resulted in improved depigmentation effects in the brown guinea pig in vivo.

\section{EXPERIMENTAL}

\section{Materials}

Arbutin (C12H16O7, MW 272.25) and polyethylene glycol 400 (PEG 400) were obtained from Sigma-Aldrich (St Louis, MO, USA). Acetonitrile (Chromasolv ${ }^{\circledR}$ HPLC) and ethanol (EtOH, Chromasolv ${ }^{\circledR}$ HPLC) were purchased from Samchun Chemical (Seoul, Republic of Korea). All chemicals were of at least analytical grade.

\section{Animals}

Seven-week-old male Hartley albino guinea pigs (Slc:Hartley) and female brownish guinea pigs (Kwl:A-1) weighing $350-400 \mathrm{~g}$ were purchased from Han-Lim (Hwaseong, Republic of Korea) and JungAng Laboratory Animals (Seoul, Republic of Korea), respectively. The animals were acclimatized for 1 week in an animal facility under controlled conditions of temperature $(23 \pm$ $\left.2{ }^{\circ} \mathrm{C}\right)$, relative humidity $(55 \pm 10 \%)$, and light (12-h light/dark, without any ultraviolet exposure). The animals had free access to laboratory diet (Purina, St. Louis, MO, USA) and ion-sterilized tap water. The animal studies were approved by the Animal Care and Use Committee of Mokpo National University (Jeonnam, Republic of Korea; Approval date, 02/05/2013; ref no., MK-05-002 for guinea pig skin permeability study and Approval date, 01/07/2013; ref no., MK-07-015 for in vivo depigmentation effect study). All experiments were performed in accordance with the Guide for the Care and Use of Laboratory Animals [15].

\section{Radiofrequency-microchanneling device}

We prepared a renovated RF-microporator composed of a reusable electronic controller and 64 disposal stainless micro-needle electrode arrays that can snap onto the end of the controller. The array was made of stainless steel electrodes $150 \mu \mathrm{m}$ in length and $250 \mu \mathrm{m}$ in diameter with a sharpened edge. The controller can measure the impedance of skin and adjust the electrical inputs during the ablation process to send the proper power to create microchannels. The following mechanical conditions were applied using the RFmicroporator: voltage; $300 \mathrm{~V}$, RF frequency; 500 $\mathrm{kHz}$, burst length; $1 \mathrm{~ms}$, output of power; $100 \%$.

\section{In vitro topical delivery of arbutin through guinea pig skin}

To confirm increased permeability of arbutin by RF microporation, dorsal skin harvested from a male Hartley albino guinea pig was used to confirm whether the permeability of arbutin increased following RF microporation. The RFmicroporator was applied five times to the outer surface of the skin $(300 \mathrm{~V} / 500 \mathrm{kHz} / 38 \mathrm{~W} / 2 \mathrm{~ms}$ per treatment) prior to the topical application of arbutin. Skin samples, either without RF treatment (control) or after RF microporation, were mounted on the receptor phase compartments with the SC facing upwards on the receptor phase compartment of a Franz diffusion cell system (Laboratory Glass Apparatus, Fine Science, Seoul, Republic of Korea) filled with 5.5 $\mathrm{mL}$ phosphate-buffered saline (PBS, $\mathrm{pH}$ 7.4). The donor compartment was clamped in place and the diffusion area was $0.785 \mathrm{~cm}^{2}$. Prior to adding the test formulations to the donor compartment, the cells were allowed to equilibrate for at least $30 \mathrm{~min}$ and the integrity of epidermal membranes were visually inspected. After equilibrium, a $200 \mu \mathrm{L}$ arbutin solution (20 $\mathrm{mg} / \mathrm{mL}$ in PEG 400-EtOH (70:30)) was placed in the donor compartments and the tops of the donor compartments were sealed with paraffin 
film to prevent sample evaporation. The receptor compartment was stirred at room temperature with a water circulating system to maintain a skin surface temperature of $32{ }^{\circ} \mathrm{C}$ throughout the experiment. Next, $200 \mu \mathrm{L}$ of the receptor phase was withdrawn after $1,3,6,9,12$, and $24 \mathrm{~h}$ and each aliquot was replaced with fresh PBS. The withdrawn samples were filtered through a membrane filter $(0.45 \mu \mathrm{m}$ polyvinylidene difluoride (PVDF), Millipore, Bedford, MA, USA) and kept at $4{ }^{\circ} \mathrm{C}$ until analysis.

The amount of arbutin retained in the skin was also determined after a $6 \mathrm{~h}$ in vitro experiment. The application site on the skin was washed 10 times using a cotton cloth immersed in distilled water. A sample of skin was weighed, cut, and homogenized in $1 \mathrm{~mL} \mathrm{EtOH}$. The supernatant containing arbutin was collected by centrifugation $\left(12,000 \times \mathrm{g}, 10 \mathrm{~min}, 4^{\circ} \mathrm{C}\right)$ and filtered through a $0.45 \mu \mathrm{m}$ PVDF membrane filter prior to further analysis. Samples from the skin-permeation experiments were quantified using an HPLC system equipped with a pre-packed C18 column (Phenomenex Gemini, $5 \mu \mathrm{m}, 250 \times 4.6 \mathrm{~mm}$ ). Aliquots $(20 \mu \mathrm{L})$ of each sample were injected into the HPLC system and arbutin was quantified at $280 \mathrm{~nm}$. The samples were chromatographed using an isocratic mobile phase consisting of monopotassium phosphate buffer (10 mM)acetonitrile $(92: 8, \mathrm{v} / \mathrm{v})$ at a flow rate of 1.0 $\mu \mathrm{L} / \mathrm{min}$. Data are expressed as the cumulative permeation amount per unit skin surface area $\left(\mu \mathrm{g} / \mathrm{cm}^{2}\right)$. Flux $\left(\mu \mathrm{g} / \mathrm{cm}^{2} \cdot \mathrm{h}\right)$ was calculated by linear regression interpolation of the experimental data.

\section{Efficacy of RF-microporated arbutin for in vivo depigmentation}

We designed a 28-day in vivo experiment to verify whether the enhanced trans-epidermal delivery of arbutin by RF microporation resulted in depigmentation. Female brown guinea pigs were exposed to UVB (total UV-irradiation energy, $800 \mathrm{~mJ} / \mathrm{cm}^{2}$ ) once per week for 3 weeks to induce hyperpigmentation on the flanks [16]. Briefly, the guinea pigs were anesthetized with pentobarbital sodium salt (35 mg/kg, P3761, Sigma, St. Louis, MO, USA), and eight separate areas $\left(1 \times 1 \mathrm{~cm}^{2}\right)$ on the left and right back of each animal were shaved and exposed to UV from five TL 20W/09N UVA Philips lamps and five TL 20 W/12RS UVB Philips lamps (Koninklijke Philips Electronics N.V., Eindhoven, The Netherlands). After the last UVB irradiation, animals were treated twice per week with or without five consecutive RF microporation treatments $(300 \mathrm{~V} / 500 \mathrm{kHz} / 38 \mathrm{~W})$ and topically painted once per day with $1 \%$ arbutin or vehicle
(PEG400:EtOH, 7:3) for 4 weeks. The L-value representing skin brightness and the $\Delta \mathrm{L}$-value representing the gap between days 0 and 7,14 , 21 and 28 were measured using a chromometer (CR-300, Minolta, Tokyo, Japan). To confirm the decrease in melanin pigments microscopically, at the end of the experiment on day 28 , the dorsal skins of animals were collected immediately after sacrifice, fixed in $10 \%$ neutral buffered formalin, processed by routine procedure, sectioned, and stained for epidermal melanin with a FontanaMasson Trichrome staining kit (KT014, DBS, CA, USA) as well as hematoxylin and eosin (H\&E) for routine evaluation.

\section{Statistical analysis}

All data are expressed as means \pm standard deviations. Statistical analysis was performed using Student's t-test to assess significant difference in in vitro skin permeability and in vivo depigmentation efficacy of arbutin between RF microporation treated and untreated group. A $p$ value of less than 0.05 was considered to be statistically significant and statistical analysis was performed using Statistical Package for the Social Sciences (SPSS) software (version 17.0, SPSS Inc., USA).

\section{RESULTS}

\section{Microchannel formation by the RF microporator}

A histological examination confirmed that the RF microporator created $50 \mu \mathrm{m}$ diameter microchannels at a depth of $100 \mu \mathrm{m}$ in the skin. $\mathrm{RF}$ microporation caused disruption of the SC but there were no significant changes in structural features of the epidermis or dermis (Figure 1).

\section{In vitro permeability through the skin of guinea pig}

The amount of accumulated arbutin was 2.25fold higher in RF-pretreated skin than in untreated skin (pretreated skin: cumulative permeation, $2288.32 \pm 280.17 \mu \mathrm{g} / \mathrm{cm}^{2}$; flux, $95.30 \pm 11.67 \mu \mathrm{g} / \mathrm{cm}^{2} \cdot \mathrm{h}$; untreated skin: cumulative permeation, $1016.67 \pm 146.55$ $\mathrm{\mu g} / \mathrm{cm}^{2}$; flux, $42.36 \pm 6.10 \mu \mathrm{g} / \mathrm{cm}^{2} \cdot \mathrm{h}$ ) (Figure $2 \mathrm{~A}$ and $B$ ). In addition, the amount of arbutin deposited in the epidermis and dermis after a $6 \mathrm{~h}$ treatment (Figure $2 \mathrm{C}$ ) was also significantly higher, being 12.59-fold higher in RF-pretreated skin $(12.20 \pm 8.23 \mu \mathrm{g} / \mathrm{mL}$ vs. $0.97 \pm 1.76 \mu \mathrm{g} / \mathrm{mL}$, $p<0.05)$, showing that skin permeability and absorption of arbutin increased due to the formation of RF microchannels. 
(A)

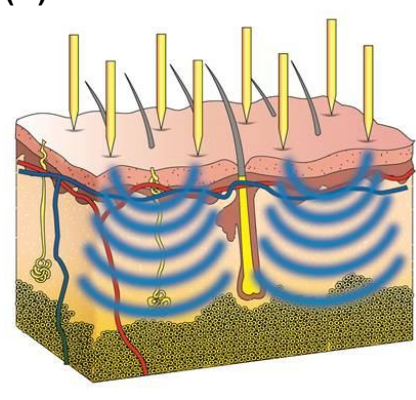

(B)

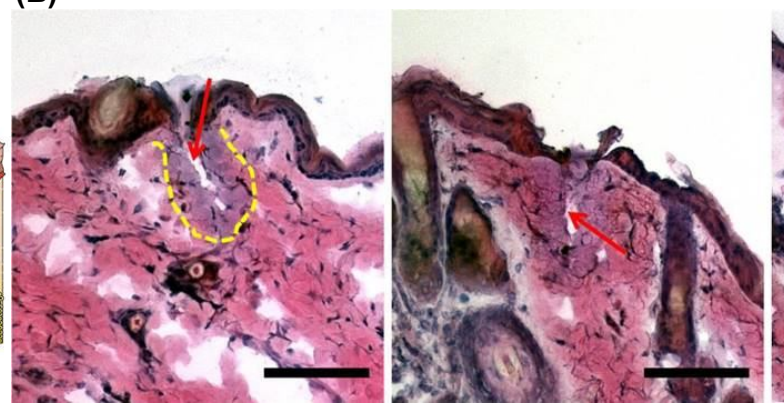

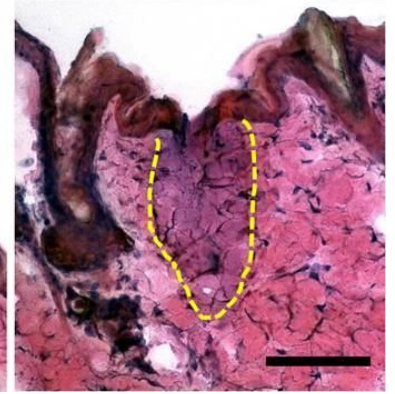

Figure 1: (A) A schematic of radiofrequency (RF) microporation. (B) Histological analysis of skin from RF microporated Hartley guinea pig skin stained with hematoxylin and eosin. Microchannels were created in the skin by RF treatment (arrows). Each microchannel was surrounded by a coagulated necrotized zone (broken lines). Bar $=100 \mu \mathrm{m}$

\section{Efficacy of RF-microporated arbutin for in vivo depigmentation}

In an in vivo study, we demonstrated that cotreatment of RF microporation and arbutin led to skin whitening. The highest $\mathrm{L}$-value and $\Delta \mathrm{L}$-value levels were noted in the UV + RF + arbutin group $(\Delta \mathrm{L}$-value on day 28 : $\mathrm{UV}, 0.17 \pm 0.50$; $\mathrm{UV}+\mathrm{RF}$, $0.07 \pm 0.48$; UV + arbutin, $1.32 \pm 0.51$ and UV + $\mathrm{RF}+$ arbutin, $1.82 \pm 0.53$, Figure $3 \mathrm{~A}$ ). No skinlightening effect was noted in the UV + RF group.

The UV + arbutin group tended to show increased $\Delta L$-values, but the result was not significant, whereas the UV + RF + arbutin group achieved a significant depigmentation effect after the 2-week treatment. Representative photographs of the treated skin agreed with the stronger depigmentation effect of $\mathrm{UV}+\mathrm{RF}+$ arbutin compared to that of UV + arbutin (Figure $3 B$ ), suggesting that RF microporation improved absorption and permeability of arbutin through the hydrophobic barrier, leading to a higher skindepigmentation effect and inhibition of melanogenesis.

Biopsy specimens were obtained after 28 days of topical application of arbutin with or without RF microporation and processed for light microscopy examination of H\&E and Fontana-Masson Trichrome stains (Figure 4). The epidermis of all animals exposed to UVB was mild to moderately hyperplastic, and keratinocytes in the upper epidermal layer exhibited small intracellular vacuoles, indicating typical mild skin irritation following UVB irradiation. However, no injured cells or infiltration of inflammatory cells were associated with the RF microporation treatment (Figure 4A). Melanin content was visualized with a Fontana-Masson Trichrome Staining kit. The quantity and distribution area of melanin decreased significantly throughout the epidermis (Figure 4B), which correlated well with the macroscopic findings, indicating that RF microporation effectively improved the permeability of arbutin against the hydrophobic barrier, leading to a greater depigmentation effect.

\section{DISCUSSION}

Micro-scale elimination of the SC layer using a device can expand the range of therapeutic molecules for topical and transdermal delivery. However, ongoing challenges include pain, high cost of the device, skin irritation or infection, and irregular microchannel formation on the skin [17]. Therefore, transdermal delivery combined with a micro-device is not appropriate for drugs with a narrow therapeutic range. In this study, we introduced an impedance feedback system to supply a constant power output, regardless of differences in skin impedance, and arrayed sharp-edged microneedles on an electrode for complete contact with the skin to create regular and reproducible microchannels.

Our results indicate that arbutin penetration through the intact skin was relatively poor due to its hydrophilic properties; however, RF microporation created a rapid increase in arbutin permeation. Alternating electrical current with a frequency $>100 \mathrm{kHz}$ caused ionic vibrations between electrodes and heated the dermis and epidermis, resulting in thermal evaporation of water, cell ablation, and removal of the SC layer. Then the microchannels were filled immediately with interstitial fluid and provided the diffusional path length in the skin necessary for arbutin permeation. For the majority of hydrophilic molecules, penetration through the SC is the rate-limiting step but the viable epidermis provides relatively favorable conditions for its diffusion. Therefore, removal of the SC layer 
(A)

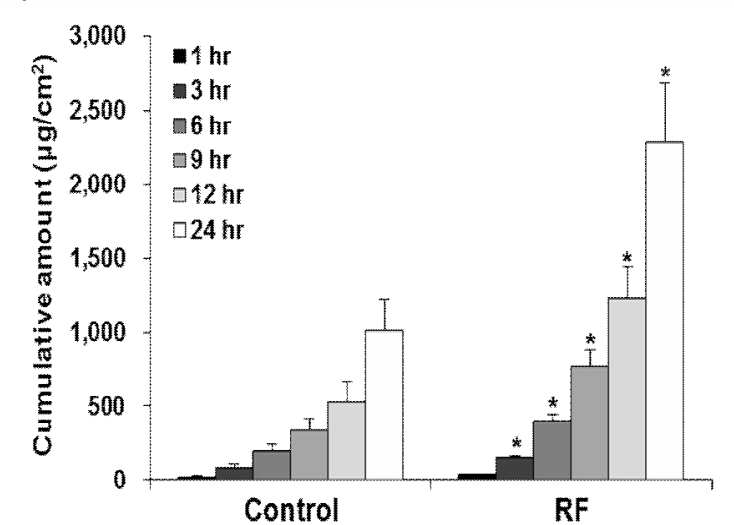

(B)

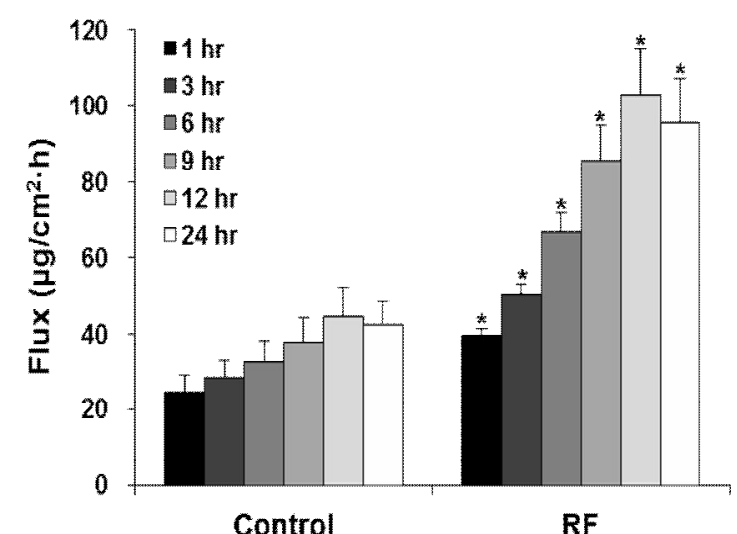

(C)

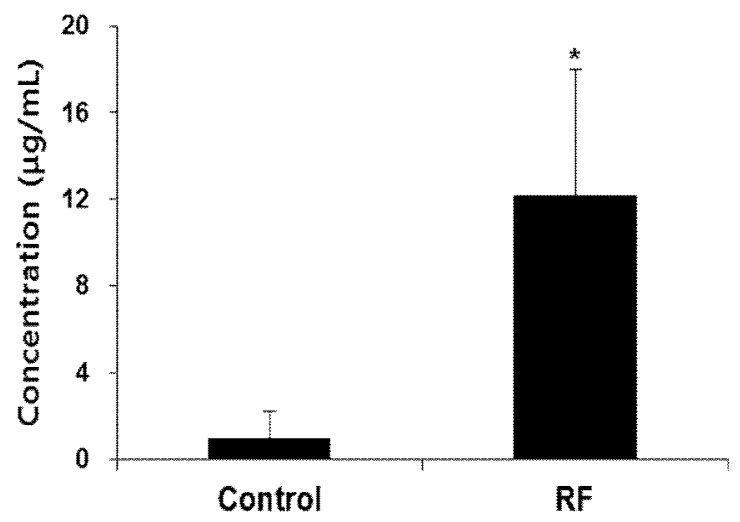

Figure 2: (A) Comparison of cumulative arbutin permeation through guinea pig skin following radiofrequency (RF) microporation. (B) In vitro fluxes of arbutin across the skin by passive diffusion and RF microporation treatment. (C) The amount of arbutin in the skin after $6 \mathrm{~h}$. RF microporation pretreatment was performed under the following conditions: applied voltage, $300 \mathrm{~V}$; RF frequency, $500 \mathrm{kHz}$; pulse time, 2 ms; number of bursts, 5; output power, $38 \mathrm{~W}$. Each value represents mean \pm standard deviation $(n=6) ;{ }^{*} p$ $<0.05$ vs. control
(A)

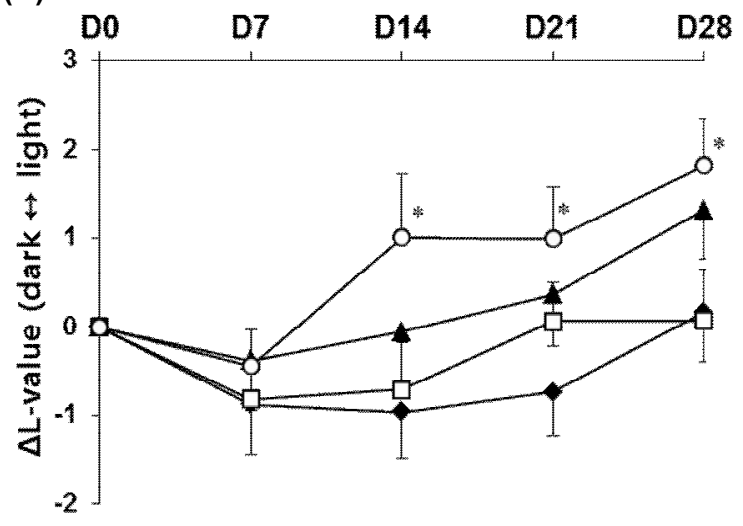

(B)

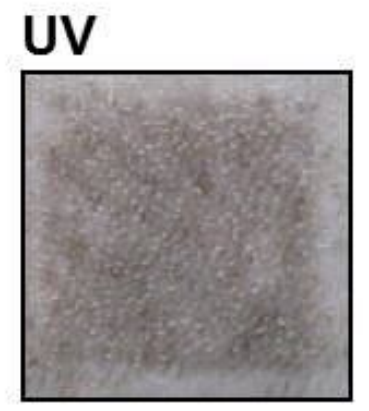

UV+RF

\section{UV+AB}

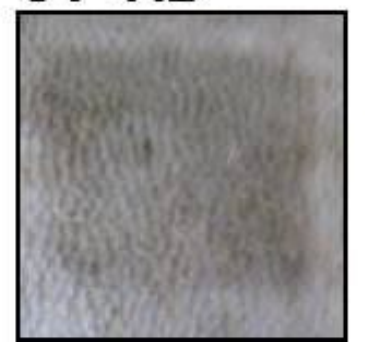

$U V+R F+A B$

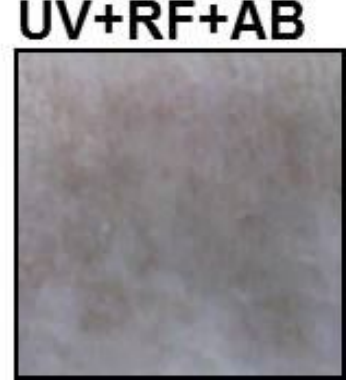

Figure 3: (A) The degree of pigmentation ( $\Delta \mathrm{L}$-value) after daily topical application of the vehicle or arbutin with or without radiofrequency (RF) microporation twice per week on UVB-induced hyperpigmentation in female brown guinea pig skin: UV + vehicle (UV, $\bullet)$, $\mathrm{UV}+\mathrm{RF}$ microporation + vehicle (UV + RF, $\square), \mathrm{UV}+$ arbutin (UV + AB, $\mathbf{\Lambda}), \mathrm{UV}+\mathrm{RF}$ microporation + arbutin (UV + RF + AB, $\circ$ ). Data are expressed as mean \pm standard deviation $(n=10) .{ }^{*} p<0.05$ vs. UV. (B) Representative images of brown guinea pig skin treated with UV + vehicle, UV + RF microporation + vehicle, UV + arbutin, UV + RF microporation + arbutin 
(A)

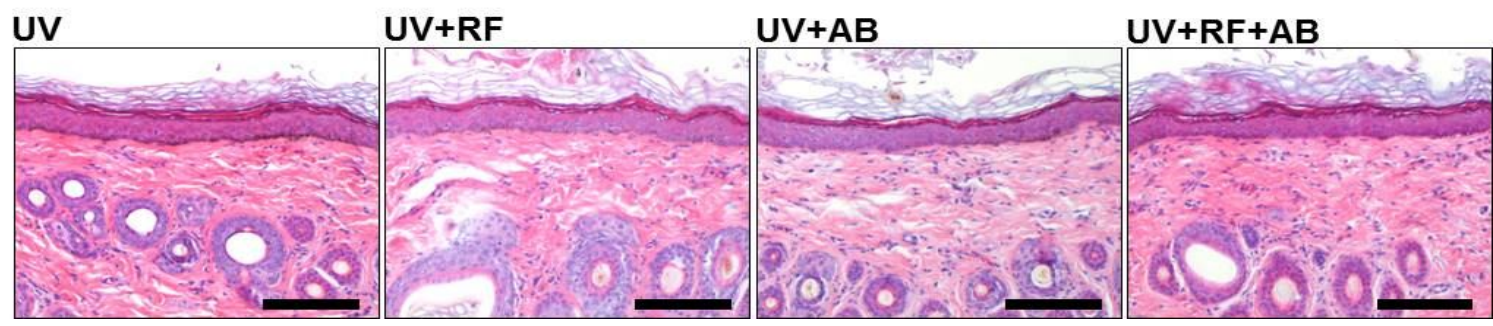

(B)

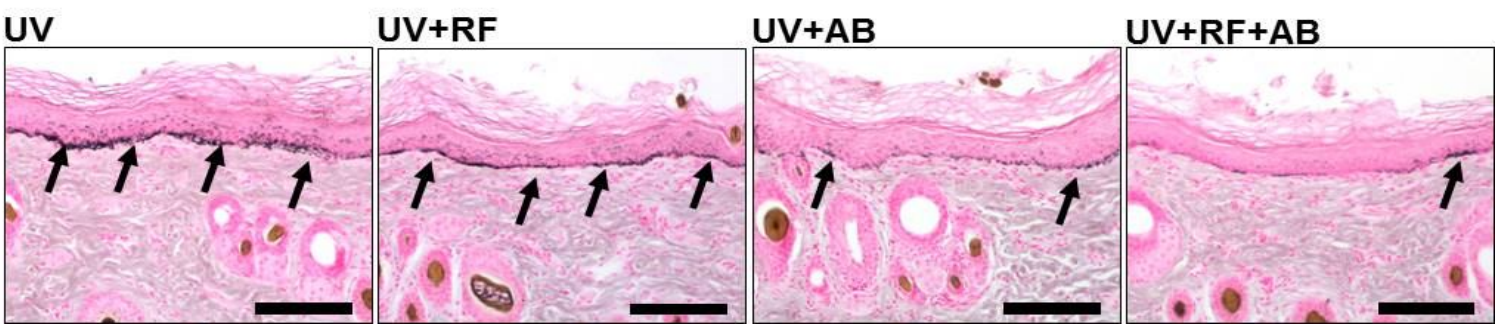

Figure 4: Biopsy specimens from the treatment site after 28 days of topical application were processed for light microscopy examination by (A) hematoxylin and eosin and (B) Fontana-Masson Trichrome staining. Black spots are melanocytes, and the number of melanocytes decreased significantly in the groups treated with radiofrequency (RF) microporation. Arrows indicate melanocyte cell bodies. Scale bars $=100 \mu \mathrm{m}$

enabled rapid delivery of arbutin through the vertical micropores followed by acceleration of lateral diffusion in the surrounding tissue.

The color of the skin is determined by epidermal melanin synthesized within melanosomes, which are specialized organelles in melanocytes [18]. Melanin synthesis is induced when skin is exposed to UV light and in medical conditions such as melasma, post-inflammatory melanoderma, and solar lentigo [19]. Although there are pharmacological and cosmetic agents that function as melanogenesis inhibitors, few are used to treat hyperpigmentation because of their clinical ineffectiveness or toxicity [20]. However, results presented in this report suggest that RF microchannel formation augmented the effective permeation of arbutin through the epidermis and dermal layers, and that the depigmentation effect was induced more quickly. Therefore, we minimized the toxic effects of various agents by reducing the treatment period or dosing amount.

\section{CONCLUSION}

The findings of this study demonstrates that RF microporation significantly improves penetration of arbutin through the skin barrier in vitro and that topical delivery of arbutin after RF pretreatment yields better skin depigmentation efficacy of photo-damaged skin in vivo. Microchannel formation through SC and epidermal layers appear to be the key factor for creating the hydrophilic pathways in the hydrophobic environment of the upper epidermis so that highly hydrophilic arbutin can be effectively delivered to the lower epidermis where melanocytes reside. Therefore, these results might serve for further applications of RF microporation technology to enhance topical and transdermal delivery of various active ingredients with high hydrophilicity.

\section{ACKNOWLEDGEMENT}

This research was supported by the Basic Science Research Program through the National Research Foundation of Korea (NRF), funded by the Ministry of Science, ICT, \& Future Planning (NRF-2014R1A1A1003122).

\section{REFERENCES}

1. Trommer $H$, Neubert $R H$. Overcoming the stratum corneum: the modulation of skin penetration. A review. Skin Pharmacol Physiol 2006; 19: 106-121.

2. Andrews S, Lee JW, Choi SO, Prausnitz MR. Transdermal insulin delivery using microdermabration. Pharm Res 2011; 28: 21102118.

3. Gustavo M, Yogeshvar NK, Richard HG. Ultrasoundenhanced transdermal transport. J Pharm Sci 2003; 92: 1125-1137.

4. Levin G, Gershonowitz A, Sacks H, Stern M, Sherman A, Rudaev S, Zivin I, Phillip M. Transdermal delivery of human growth hormone through RF-microchannels. Pharm Res 2005; 22: 550-555. 
5. Nelson JS, McCullough JL, Glenn TC, Wright WH, Liaw $L H$, Jacques SL. Midinfrared laser ablation of stratum corneum enhanced in vitro percutaneous transport of drugs. J Invest Dermatol 1991; 97: 874-879.

6. Singh TR, Garland MJ, Cassidy CM, Migalska K, Demir YK, Abdelghany S, Ryan E, Woolfson AD, Donnelly RF. Microporation techniques for enhanced delivery of therapeutic agents. Recent Pat Drug Deliv Formul 2010; 4: 1-17.

7. Otto G, Düber C, Hoppe-Lotichius M, König J, Heise M, Pitton MB. Radiofrequency ablation as first-line treatment in patients with early colorectal liver metastases amenable to surgery. Ann Surg 2010; 251: 796-803.

8. Hruza G, Taub AF, Collier SL, Mulholland SR. Skin rejuvenation and wrinkle reduction using a fractional radiofrequency system. J Drugs Dermatol 2009; 8: 259-265.

9. Tsai TH, Lin SJ, Lee WR, Wang CC, Hsu CT, Chu T, Dong $C Y$. Visualizing radiofrequency-skin interaction using multiphoton microscopy in vivo. J Dermatol Sci 2012; 65: 95-101.

10. Birchall J, Coulman S, Anstey A, Gateley C, Sweetland $H$, Gershonowitz A, Neville L, Levin G. Cutaneous gene expression of plasmid DNA in excised human skin following delivery via microchannels created by radio frequency ablation. Int J Pharm 2006; 312: 1523.

11. Sintov AC, Krymberk I, Daniel D, Hannan T, Sohn Z, Levin G. Radiofrequency-driven skin microchanneling as a new way for electrically assisted transdermal delivery of hydrophilic drug. J Control Release 2003; 29: $311-320$.
12. O'Donoghue JL. Hydroquinone and its analogues in dermatology - a risk-benefit viewpoint. J Cosmet Dermatol 2006; 5: 196-203.

13. Sugimoto $K$, Nishimura $T$, Nomura $K$, Sugimoto $K$, Kuriki T. Inhibitory effects of alpha-arbutin on melanin synthesis in cultured human melanoma cells and a three-dimensional human skin model. Biol Pharm Bull 2004; 27: 510-514.

14. Som I, Bhatia K, Yasir M. Status of surfactants as penetration enhancers in transdermal drug delivery. $J$ Pharm Bioallied Sci 2012; 4: 2-9.

15. National Research Council of the National Academies. Guide for the care and use of laboratory animals. 8th ed. Washington DC: National Academies Press; 2011.

16. Hwang JS, Lee HY, Lim TY, Kim MY, Yoon TJ. Disruption of tyrosinase glycosylation by $\mathrm{N}$ acetylglucosamine and its depigmenting effects in guinea pig skin and in human skin. J Dermatol Sci 2011; 63: 199-201.

17. Arora A, Prausnitz MR, Mitragotri S. Micro-scale devices for transdermal drug delivery. Int J Pharm 2008; 364: 227-236.

18. Hearing VJ, Tsukamoto K. Enzymatic control of pigmentation in mammals. FASEB J 1991; 5: 29022909.

19. Gilchrest BA, Park HY, Eller MS, Yaar M. Mechanisms of ultraviolet light-induced pigmentation. Photochem Photobiol 1996; 63: 1-10.

20. Briganti S, Camera E, Picardo M. Chemical and instrumental approaches to treat hyperpigmentation. Pigment Cell Res 2003; 16: 101-110. 